\title{
Graph-Based Clustering of Random Point Set
}

\author{
Atsushi Imiya ${ }^{1}$ and Ken Tatara ${ }^{2}$ \\ 1 Institute of Media and Information Technology, Chiba University, \\ Yayoi-cho 1-33, Inage-ku, 263-8522, Chiba Japan \\ 2 School of Science and Technology, Chiba University, \\ Yayoi-cho 1-33, Inage-ku, 263-8522, Chiba Japan
}

\begin{abstract}
In this paper, we define clusters and the boundary curves of clusters in a random point set using the Delaunay triangulation and the principal curve analysis. The principal curve analysis is a generalization of principal axis analysis, which is a standard method for data analysis in pattern recognition.
\end{abstract}

\section{Introduction}

In this paper, we develop a graph-based algorithm for clustering of point sets and learning of the boundary of random point sets. The boundary of a random point set is extracted by the principal curve analysis. The principal curve analysis is a generalization of principal axis analysis, which is a standard method for data analysis in pattern recognition.

For the vector space method of data mining, each datum is expressed as a point in the higher dimensional Euclidean space. Symbolic expressions of these point sets are required for the visual interface for the data mining systems. Furthermore, these data are sometimes transformed as a point distribution in lower dimensional vector spaces, usually tow or three dimensional spaces, for the visualisation of data distribution on CRT. Therefore, the extraction of the symbolic features of random point sets in two and three dimensional is a basic process for the visual interpretation of random point sets for the visualisation of the data space.

Computational geometry provides combinatorial methods for the recovery of boundary curves as polygonal curves. These algorithms are based on Voronoi tessellation, Delaunay triangulation, Gabriel graphs, crust, $\alpha$-shape, and $\beta$-skeleton $[1-3]$. The reconstructed curves by these methods are piecewise linear. Furthermore, the solutions are sensitive against noise and outlayers, since these methods construct polygons and polyhedrons using all sample points. Furthermore, the algorithm extracts a boundary curves.

In this paper, we introduce method for the estimation of the boundary of a random point set which permits the extraction of boundary curves of clusters in a random point set. 


\section{Mathematical Preliminary}

Delaunay Triangulation. Setting $\left\{\boldsymbol{p}_{i}\right\}_{i=1}^{n}$ to be a point set in $\mathbf{R}^{n}$, the region

$$
\mathbf{V}_{i}=\left\{\boldsymbol{x}|| \boldsymbol{x}-\boldsymbol{p}_{\boldsymbol{i}}|\leq| \boldsymbol{x}-\boldsymbol{p}_{j} \mid, i \neq j\right\}
$$

is called Voronoi region with respect to the generator $\boldsymbol{p}_{i}$. The hyperplane

$$
F_{i}=\left\{\boldsymbol{x}|| \boldsymbol{x}-\boldsymbol{p}_{\boldsymbol{i}}|=| \boldsymbol{x}-\boldsymbol{p}_{j} \mid\right\}
$$

is the Voronoi face. Setting $\boldsymbol{p}_{i}$ and $\boldsymbol{p}_{j}$ to be the generators of Voronoi regions sharing a face, a geometric graph which connect all pairs of generators in the face-sharing region is called Delaunay triangulation. The Voronoi tessellation and the Delaunay triangulation are dual figures each other.

Mathematical Morphology. Setting $\mathbf{A}$ to be a finite closed set in the $n$ dimensional Euclidean space $\mathbf{R}^{n}$, the Minkowski addition and subtraction of sets are defined as

$$
\mathbf{A} \oplus \mathbf{B}=\bigcup_{\boldsymbol{x} \in \mathbf{B}, \boldsymbol{y} \in \mathbf{B}}(\boldsymbol{x}+\boldsymbol{y}), \quad \mathbf{A} \ominus \mathbf{B}=\overline{\mathbf{A} \oplus \overline{\mathbf{B}}} .
$$

The inner and outer boundary of point set $\mathbf{A}$ with respect to radius $\lambda$ are defined as

$$
\Delta_{\lambda}^{+} \mathbf{A}=(\mathbf{A} \oplus \lambda \boldsymbol{B}) \backslash \mathbf{A}, \Delta_{\lambda}^{-} \mathbf{A}=\mathbf{A} \backslash(\mathbf{A} \ominus \lambda \boldsymbol{B})
$$

for the unit $n$-sphere such that $\boldsymbol{B}=\{\boldsymbol{x} \mid \boldsymbol{x} \leq 1\}$, where $\lambda \mathbf{B}=\{\lambda \boldsymbol{x} \mid \boldsymbol{x} \in \mathbf{B}\}$ for $\lambda>0$. We call $\mathbf{A}_{\lambda}=\Delta_{\lambda}^{+} \mathbf{A} \bigcup \Delta_{\lambda}^{-} \mathbf{A}$ the boundary belt of $\mathbf{A}$ with respect to $\lambda$. Geometrically, we have the relation

$$
\lim _{\lambda \rightarrow+0} \mathbf{A}_{\lambda}=\partial \mathbf{A},
$$

where $\partial \mathbf{A}$ is the boundary curve of set $\mathbf{A}$.

Principal Curve Analysis. Let $\mathbf{X}$ be a mean-zero point distribution in $\mathbf{R}^{n}$. The major principal component $\boldsymbol{w}$ maximizes the criterion

$$
J(\boldsymbol{w})=E_{\boldsymbol{x} \in \mathbf{X}}\left|\boldsymbol{x}^{\top} \boldsymbol{w}\right|^{2}
$$

with respect to $|\boldsymbol{w}|=1$, where $E_{\boldsymbol{x} \in \mathbf{X}}$ expresses the expectation over set $\mathbf{X}$. Line $\boldsymbol{x}=t \boldsymbol{w}$ is a one-dimensional linear subspace which approximates $\mathbf{X}$. A maximization criterion

$$
J(\boldsymbol{P})=E \boldsymbol{x} \in \mathbf{X}|\boldsymbol{P} \boldsymbol{x}|^{2}
$$

with respect to $\operatorname{rank} \boldsymbol{P}=1$, determines a one dimensional linear subspace which approximates $\mathbf{X}$. If $\mathbf{X}$ is not a mean-zero point distribution in $\mathbf{R}^{2}$ and the centroid of $\mathbf{X}$ is not predetermined, the maximization criterion

$$
J(\boldsymbol{P}, \boldsymbol{g})=E_{\boldsymbol{x} \in \mathbf{X}}|\boldsymbol{P}(\boldsymbol{x}-\boldsymbol{g})|^{2}
$$


with respect to $\operatorname{rank} \boldsymbol{P}=1$, determines a one-dimensional linear manifold which approximates point distribution $\mathbf{X}$.

For the partition of $\mathbf{X}$ into $\left\{\mathbf{X}_{i}\right\}_{i=1}^{N}$ such that $\mathbf{X}=\cup_{i=1}^{N} \mathbf{X}_{i}$, vectors $\boldsymbol{g}_{i}$ and $\boldsymbol{w}_{i}$ which maximize the criterion

$$
J\left(\boldsymbol{w}_{1}, \cdots, \boldsymbol{w}_{N}, \boldsymbol{g}_{1}, \cdots, \boldsymbol{g}_{N}\right)=\sum_{i=1}^{N} E_{\boldsymbol{x} \in \mathbf{X}_{i}}\left|\left(\boldsymbol{x}-\boldsymbol{g}_{i}\right)^{\top} \boldsymbol{w}_{i}\right|^{2}
$$

determine a polygonal curve [4],

$$
\boldsymbol{l}=\boldsymbol{g}_{i}+t \boldsymbol{w}_{i}
$$

Furthermore, for an appropriate partition of $\mathbf{X}$ into $\{\mathbf{X}\}_{i=1}^{N}$, such that $\mathbf{X}=$ $\cup_{i=1}^{N} \mathbf{X}_{i}$, vector $\boldsymbol{g}_{i}$ and orthogonal projector $\boldsymbol{P}_{i}$, which maximize the criterion

$$
J\left(\boldsymbol{P}_{1}, \cdots, \boldsymbol{P}_{N}, \boldsymbol{g}_{1}, \cdots, \boldsymbol{g}_{N}\right)=\sum_{i=1}^{N} E_{\boldsymbol{x} \in \mathbf{X}_{i}}\left|\boldsymbol{P}_{i}\left(\boldsymbol{x}-\boldsymbol{g}_{i}\right)\right|^{2}
$$

with respect to $\operatorname{rank} \boldsymbol{P}_{i}=1$, determine a piecewise linear curve,

$$
\mathbf{C}_{i}=\left\{\boldsymbol{x}+\boldsymbol{g}_{i} \mid \boldsymbol{P}_{i} \boldsymbol{x}=\boldsymbol{x}\right\} .
$$

This piecewise linear is called the principal curve [4]. Figure 1 shows the principal components and principal curves on a plane.

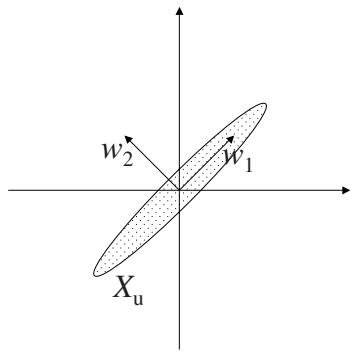

(a)

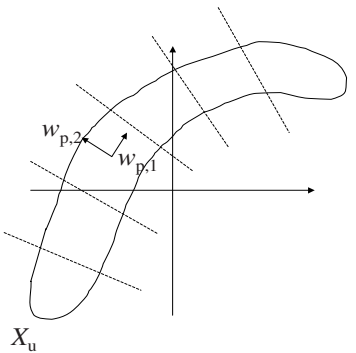

(b)

Fig. 1. Principal components (a) and Principal curve (b).

\section{Graph-Based Clustering}

Using Delaunay triangulation of a random point set, we develop an algorithm for the separation of clusters of point set. We assume that in each subset, the distances between two points connected by a Delaunay edge is shorter than a constant. We call this constant the resolution of point sets. We adopt the median of Delaunay edges as the estimation of the resolution of the point sets. 
Definition 1 For a point $\boldsymbol{p}$ in a random point set $\mathbf{V}$, we call

$$
\boldsymbol{p}_{\delta}=\left\{\boldsymbol{x}|| \boldsymbol{p}-\boldsymbol{x} \mid<\delta, \boldsymbol{p} \in \mathbf{V}, \forall \boldsymbol{x} \in \mathbf{R}^{2}\right\}
$$

the effective region of point $\boldsymbol{p}$ with respect to radius $\delta$.

As the union of the effective region of each point, we define the effective region of a random point set.

Definition 2 For a random point set $\mathbf{V}$, we call

$$
\overline{\mathbf{V}}=\bigcup_{\boldsymbol{p} \in \mathrm{V}} \boldsymbol{p}_{\delta}
$$

the effective region of point set $\mathbf{V}$ with respect to radius $\delta$.

If points in $\mathbf{V}$ are sampled from a connected region in $\mathbf{R}^{n}, \overline{\boldsymbol{V}}$ becomes a connected region in $\mathbf{R}^{n}$, selecting an appropriate $\delta$. Therefore, we introduce a method for the selection of a suitable radius for the estimation of the connected region from a random point set. Using this estimated connected region, we develop an algorithm for the construction of the boundary of a random point set.

Setting $E$ to be the set of edges of the Delaunay triangulation $D$ constructed from the points in random point set $\mathbf{V}$, we set

$$
\delta=\operatorname{median}_{e \in E}|e|,
$$

if points distribute uniformly in a region. Then, we define the boundary set as

$$
\mathbf{V}_{\gamma}=\overline{\mathbf{V}}_{\gamma} \bigcap \mathbf{V}, \overline{\mathbf{V}}_{\gamma}=\overline{\mathbf{V}} \backslash\{\overline{\mathbf{V}} \ominus \gamma \boldsymbol{D}(\delta)\}
$$

where $\gamma>1$ is a constant and $\boldsymbol{D}=\{\boldsymbol{x}|| \boldsymbol{x} \mid \leq \delta\}$ is the set of all points in the circle with radius $\delta$. We call $\mathbf{V}_{\gamma}$ the $\gamma$-boundary of random point set $\mathbf{V}$.

Next, we construct the new Delaunay triangulation $D^{\prime}$ for points in

$$
\mathbf{V}^{\prime}=\mathbf{V} \oplus \delta\left\{\left\{\boldsymbol{d}_{i}\right\}_{i=1}^{n}\right\}
$$

where $\boldsymbol{d}_{i}$ is an appropriate vector such that $\left|\boldsymbol{d}_{i}\right|=1$. We call the point set $\mathbf{V}^{\prime}$ the effective set of $\mathbf{V}$.

After Delaunay triangulation $D^{\prime}$ of the new point set, there exist three types of edges as shown in Figure 2 (e), edges connect points in $\mathbf{V}$, edges connect points in $\mathbf{V}^{\prime}$, and edges connect points in $\mathbf{V}$ and $\mathbf{V}^{\prime}$. As shown in (a), in the neighborhood of points in each cluster of $\mathbf{V}$, there exist points in $\mathbf{V}^{\prime}$, since points in $\mathbf{V}^{\prime} \backslash \mathbf{V}$ lie in the region $D(\delta) \oplus \boldsymbol{p}_{i}$ for a point $\boldsymbol{p}_{i} \in \boldsymbol{V}$. This geometrical property leads to the conclusion that in Delaunay triangulation $D^{\prime}$, edges which connect points in $\mathbf{V}$ is the bridges which connect clusters. Therefore, we have the following clustering algorithm using Delaunay triangulation.

1. Construct Delaunay triangulation $D$ from random point set $\mathbf{V}$.

2. Assign label 1 to points in the random point set $\mathbf{V}$. 
3. For the collection of all edges $E$ of $D$, detect the median length, and set it as $\delta$.

4. Construct $\mathbf{V}^{\prime}=\mathbf{V} \oplus \delta\left\{\left\{\boldsymbol{d}_{i}\right\}_{i=1}^{n}\right\}$, for appropriate unit vectors $\left\{\boldsymbol{d}_{i}\right\}_{i=1}^{n}$

5. Assign label $\mathbf{2}$ to point in $\mathbf{V}^{\prime}$.

6. Construct Delaunay $D^{\prime}$ from $\mathbf{V}^{\prime}$.

7. Eliminate edges connect whose both vertices are labelled as $\mathbf{1}$.

Figure 2 shows a process for the clustering. Delaunay triangulation (b) of the original point set (a) determines the effective region (c). After Delaunay triangulation (e) of point in the effective set (d), the algorithm extract bridges (f). By eliminating bridges (g) and (h), the algorithm yields clusters.

\section{Detection of Cluster Boundary}

After extracting clusters from random point set, we extract the boundary of each cluster using the principal curve analysis. As the boundary of each cluster, we extract the principal curve from the $\gamma$-boundary.

Definition 3 The principal boundary of a random point set is the principal manifold of the point in the $\gamma$-boundary of a random point set.

We also call this principal manifold extracted from random point set $\mathbf{V}$ the $\gamma$-curve of $\mathbf{V}$.

\subsection{Principal Curves Detection}

Set $\mathbf{D}$ and $\mathbf{S}$ to be a random point set and the vertices of polygonal curve, respectively, and the distance between point $\boldsymbol{x} \in \mathbf{S}$ and $\boldsymbol{y} \in \mathbf{D}$ is defined as $d(\boldsymbol{x}, \mathbf{D})=\min \boldsymbol{y} \in \mathbf{D} d(\boldsymbol{x}, \boldsymbol{y})$ for the Euclidean distance in a plane.

The initial shapes $\mathbf{S}$ and $\mathbf{C}$ are a line segment whose direction is equivalent to the major component $\boldsymbol{w}_{1}$ of a random point set and a regular triangle whose vertices are determined from the principal components $\boldsymbol{w}_{1}$ and $\boldsymbol{w}_{2}$. For a sequence of vertices $\left\langle\boldsymbol{v}_{1}, \boldsymbol{v}_{2}, \cdots \boldsymbol{v}_{n}\right\rangle$ of a polygonal curve, we define the tessellation as

$$
\begin{aligned}
V_{\alpha} & =\left\{\boldsymbol{x} \mid d\left(\boldsymbol{x}, \boldsymbol{v}_{\alpha}\right)<d\left(\boldsymbol{x}, \boldsymbol{v}_{i}\right), d\left(\boldsymbol{x}, \boldsymbol{v}_{\alpha}\right)<d\left(\boldsymbol{x}, \boldsymbol{e}_{i j}\right), \alpha \neq i\right\}, \\
E_{\alpha \alpha+1} & =\left\{\boldsymbol{x} \mid d\left(\boldsymbol{x}, \boldsymbol{e}_{\alpha \alpha+1}\right)<d\left(\boldsymbol{x}, \boldsymbol{v}_{i}\right), d\left(\boldsymbol{x}, \boldsymbol{e}_{\alpha \alpha+1}\right)<d\left(\boldsymbol{x}, \boldsymbol{e}_{i i+1}\right), \alpha \neq i\right\},
\end{aligned}
$$

where $\boldsymbol{e}_{i i+1}$ is the edge which connects $\boldsymbol{v}_{i}$ and $\boldsymbol{v}_{i+1}$. The minimization criterion of reference [5] is expressed as

$$
I=\sum_{\boldsymbol{v}_{k} \in \mathbf{C}} F\left(\boldsymbol{v}_{k}, \mathbf{D}\right)+\lambda \sum_{\boldsymbol{v}_{k} \in \mathbf{P}} \sum_{i=-1}^{1} \frac{\boldsymbol{v}_{i-1 i}^{\top} \boldsymbol{v}_{i i+1}}{\left|\boldsymbol{v}_{i-1 i}\right|\left|\boldsymbol{v}_{i i+1}\right|}
$$

for

$$
F\left(\boldsymbol{v}_{k}, \mathbf{D}\right)=\sum_{\boldsymbol{x} \in E_{k-1 k}} d\left(\boldsymbol{x}, \boldsymbol{v}_{k}\right)+\sum_{\boldsymbol{x} \in V_{k}} d\left(\boldsymbol{x}, \boldsymbol{v}_{k}\right)+\sum_{\boldsymbol{x} \in E_{k k+1}} d\left(\boldsymbol{x}, \boldsymbol{v}_{k}\right) .
$$

Using this criterion, we obtain an algorithm for the detection of the principal curve [5] where $I_{K}$ is the value of $I$ with $K$ vertices. 


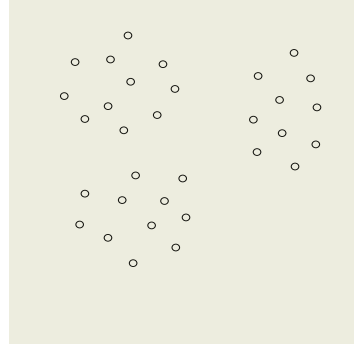

(a)

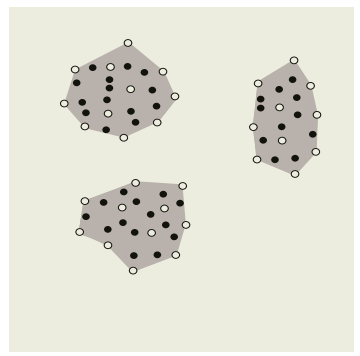

(d)

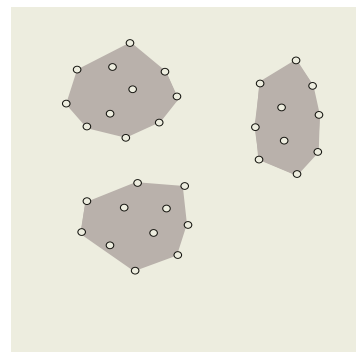

(g)

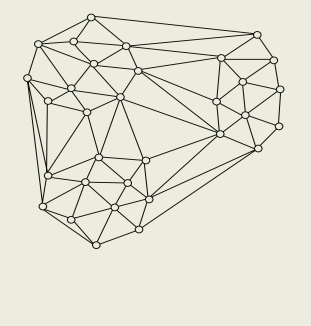

(b)

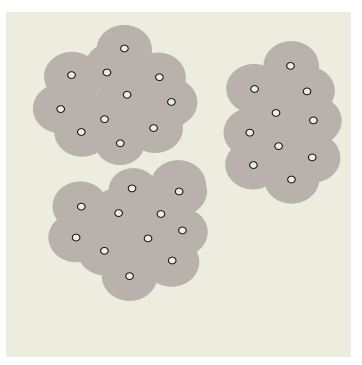

(c)

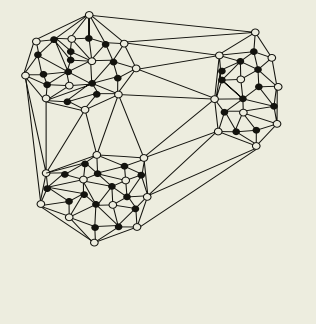

(e)

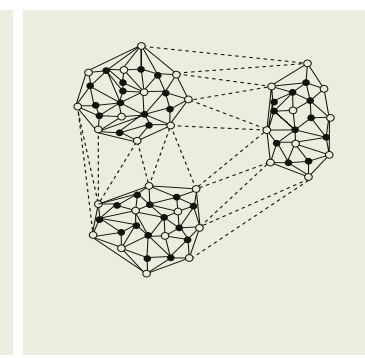

(f)

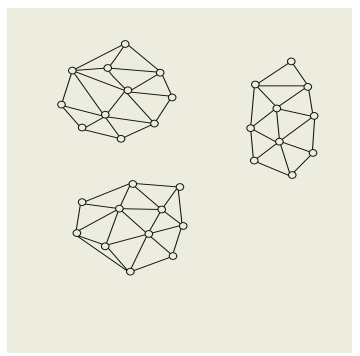

(h)

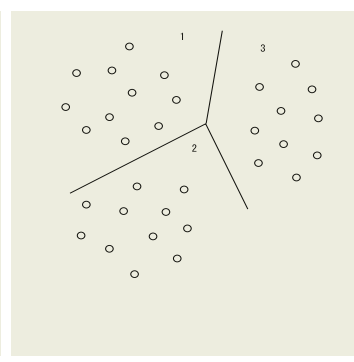

(i)

Fig. 2. Clustering algorithm: Delaunay triangulation (b) of the original point set (a) determines the effective region (c). After Delaunay triangulation (e) of point in the effective set (d), the algorithm extract bridges (f). By eliminating bridges (g) and (h), the algorithm yields clusters.

1. Set the vertices of the initial curve as $\mathbf{S}$.

2. Move all vertices $\boldsymbol{v}_{i} i=1,2, \cdots, K$, to minimize $I_{K}$.

3. Generate the new vertex $\boldsymbol{v}_{K+1}$ on the curve $\mathbf{S}$.

4. If $\left|I_{K}-I_{K-1}\right| \leq \varepsilon$ for a positive constant $\varepsilon$, then stop, else set $\mathbf{S}:=\mathbf{S} \cup\left\{\boldsymbol{v}_{K+1}\right\}$ and go to 2 . 
This incremental algorithm preserves the topology of the initial curve, since the algorithm generates new vertices on the curve.

\subsection{Boundary Curve Detection}

It is possible to detect boundary of random point set if we can extract the boundary belt of random point set. In this section, we develop an algorithm for the extraction of the boundary belt.

Using definitions in the previous sections, we have the following algorithm for the construction of the principal boundary of a random point set.

1. Construct Delaunay triangulation $D$ from random point set $\mathbf{V}$.

2. For the collection of all edges $E$ of $D$, detect the median length, and set it as $\delta$.

3. Compute the effective region of random point set $\mathbf{V}$.

4. Compute $\gamma$-boundary of random point set $\mathbf{V}$.

5. Compute $\gamma$-curve of random point set $\mathbf{V}$.

The construction of the Delaunay triangulation using all points in $\mathbf{V}$ is in practice an time-consuming process for a large number of points even if we use an optimal algorithm. Furthermore, we only need the lengths of the Delaunay triangles for the construction of the effective region of the neighborhood of a random point set. Therefore, we replace steps 1 and 2 of the algorithm to the following random sampling process.

1. Select a finite closed subset $\mathbf{S}$ of $\mathbf{R}^{2}$.

2. Compute Delaunay triangulation for points in $\mathbf{S} \cap \mathbf{V}$.

3. Compute the median of length of edges of Delaunay triangles with respect to subset $\mathbf{S}$.

4. Repeat steps 1 to 3 until the predetermined number of times.

5. Select the maximum length.

In Figure 3, we show, the sequence of boundary curves of a sequence of random point sets which changes number of clusters. The clusters are generated using geometric property of the Delaunay edges.

Figure 4, we also show an example of clustering. 4299 points in (a) are separated to three clusters which are encircled by curves in (b).

In these examples, the density of point distribution are $0.3 /$ unit length $^{2}$. Therefore, the average distance between points are 3 to 4 units. Furthermore, the median of the edges of the first Delaunay triangulation are 4 to 5 units. These results allow that the median of the lengths the edges of the first Delaunay triangulation is a reasonable estimation of the resolution of the random point sets. 


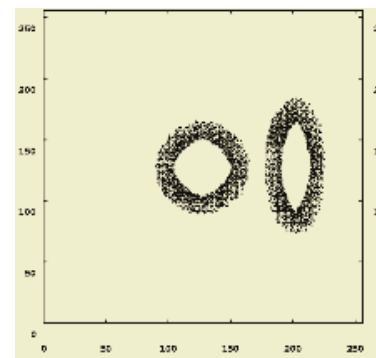

(a)

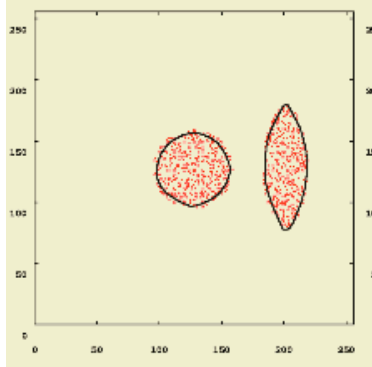

(d)

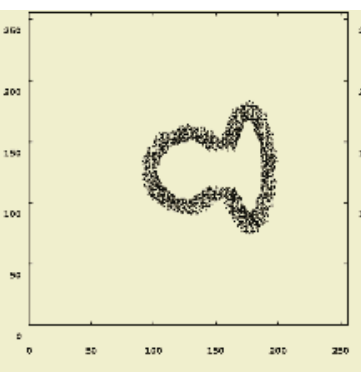

(b)

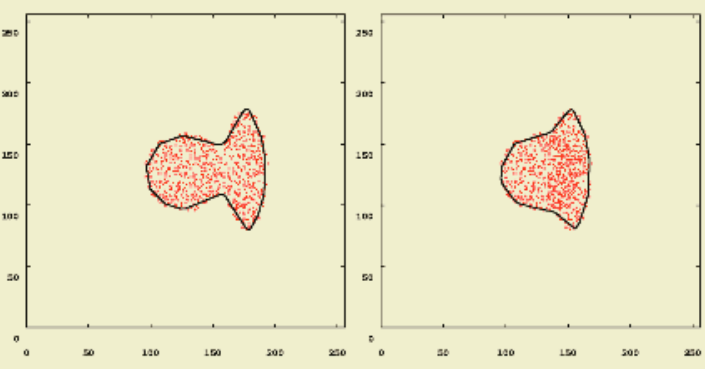

(e)

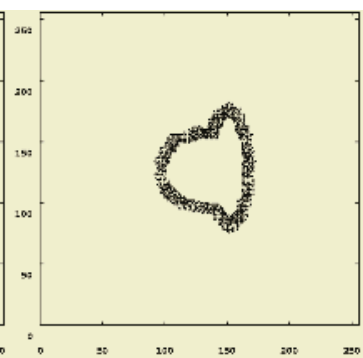

(c)

Fig. 3. The sequence of boundary belts (a), (b), and (c) and curves (d), (e), (f) of a sequence of random point sets which changes number of clusters. The clusters are generated using geometric property of the Delaunay edges.

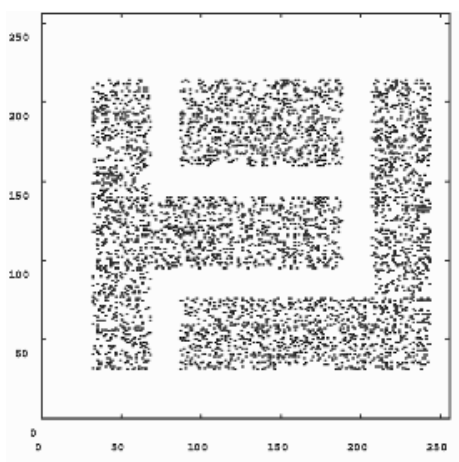

(a)

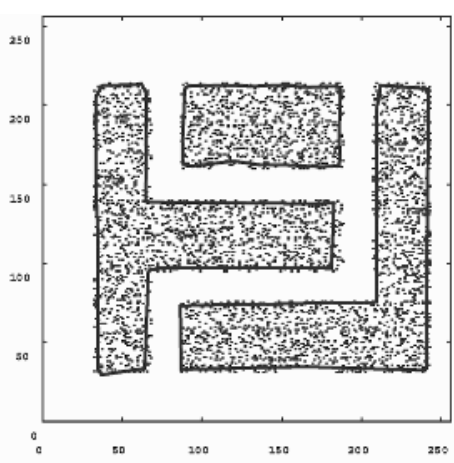

(b)

Fig. 4. An example of clustering. 4299 points in (a) are separated to three clusters which are encircled by curves in (b). 


\section{Conclusions}

In this paper, we have defined clusters and the boundary curves of clusters in a random point set using the Delaunay triangulation and the principal curve analysis. Once the polygonal boundary of a random point set is estimated, it is possible to compute the linear skeleton of the polygonal boundary $[10,11]$. We adopt the linear skeleton of the polygonal boundary as the skeleton of a random point set.

\section{References}

1. Amenta, N., Bern, M., and Eppstein, D., The crust and the $\beta$ skeleton:Combinatorial curve reconstruction, Graphical Models and Image Processing, 60, 125-135, 1998.

2. Attali, D. and Montanvert, A., Computing and simplifying 2D and 3D continuous skeletons, CVIU, 67, 261-273, 1997.

3. Edelsbrunner, H., Shape reconstruction with Delaunay complex, Lecture Notes in Computer Science, 1380, 119-132, 1998.

4. Hasite, T., Stuetzle, T., Principal curves, J. Am. Statistical Assoc., 84, 502-516, 1989.

5. Kégl, B., Krzyzak, A., Linder, T., Zeger, K., Learning and design of principal curves, IEEE PAMI, 22, 281-297, 2000.

6. Oja, E., Principal components, minor components, and linear neural networks, Neural Networks, 5, 927-935, 1992.

7. Imiya, A., Ootani, H., Kawamoto, K., Linear manifolds analysis: Theory and algorithm, Neurocomputing, 57, 171-187, 2004.

8. Imiya, A., Kawamoto, K., Learning dimensionality and orientations of 3D objects, Pattern Recognition Letters, 22, 75-83, 2001.

9. Silverman, B. W., Some aspects of the spline smoothing approach to nonparametric regression curve fitting, J. R. Statist. Soc, B. 47, 1-52, 1985.

10. Bookstein, F. L., The line-skeleton, CVGIP, 11, 1233-137, 1979

11. Rosenfeld, A., Axial representations of shapes, CVGIP, 33, 156-173, 1986. 